\title{
Impact of Preoperative Opioid Use on Patient-Reported Outcomes after Revision Total Knee Arthroplasty: A Propensity Matched Analysis
}

\author{
Eitan Ingall, MD ${ }^{1}$ Christian Klemt, $\mathrm{PhD}^{1} \quad$ Christopher M. Melnic, MD ${ }^{1}$ Wayne B. Cohen-Levy, MD ${ }^{1}$ \\ Venkatsaiakhil Tirumala, $\mathrm{MSc}^{1}$ Young-Min Kwon, MD, $\mathrm{PhD}^{1}$
}

\footnotetext{
${ }^{1}$ Department of Orthopaedic Surgery, Bioengineering Laboratory, Massachusetts General Hospital, Harvard Medical School, Boston, Massachusetts
}

J Knee Surg 2023;36:115-120.
Address for correspondence Young-Min Kwon, MD, PhD, Department of Orthopaedic Surgery, Bioengineering Laboratory, Massachusetts General Hospital, Harvard Medical School, 55 Fruit Street, Boston, MA 02114 (e-mail: ymkwon@mgh.harvard.edu).

\begin{abstract}
Keywords

- opioid

- patient-reported outcomes measures

- revision knee arthroplasty

This is a retrospective study. Prior studies have characterized the deleterious effects of narcotic use in patients undergoing primary total knee arthroplasty (TKA). While there is an increasing revision arthroplasty burden, data on the effect of narcotic use in the revision surgery setting remain limited. Our aim was to characterize the effect of active narcotic use at the time of revision TKA on patient-reported outcome measures (PROMs). A total of 330 consecutive patients who underwent revision TKA and completed both pre- and postoperative PROMs was identified. Due to differences in baseline characteristics, 99 opioid users were matched to 198 nonusers using the nearest-neighbor propensity score matching. Pre- and postoperative knee disability and osteoarthritis outcome score physical function (KOOS-PS), patient reported outcomes measurement information system short form (PROMIS SF) physical, PROMIS SF mental, and physical SF 10A scores were evaluated. Opioid use was identified by the medication reconciliation on the day of surgery. Propensity score-matched opioid users had significantly lower preoperative PROMs than the nonuser for KOOS-PS (45.2 vs. 53.8, $p<0.01$ ), PROMIS SF physical ( 37.2 vs. $42.5, p<0.01$ ), PROMIS SF mental (44.2 vs. $51.3, p<0.01$ ), and physical SF $10 \mathrm{~A}$ (34.1 vs. $36.8, p<0.01$ ). Postoperatively, opioid-users demonstrated significantly lower scores across all PROMs: KOOS-PS (59.2 vs. 67.2, $p<0.001$ ), PROMIS SF physical (43.2 vs. 52.4, $p<0.001$ ), PROMIS SF mental (47.5 vs. $58.9, p<0.001)$, and physical SF $10 \mathrm{~A}(40.5$ vs. $49.4, p<0.001)$. Propensity score-matched opioid-users demonstrated a significantly smaller absolute increase in scores for PROMIS SF Physical $(p=0.03)$ and Physical SF 10A $(p<0.01)$, as well as an increased hospital length of stay $(p=0.04)$. Patients who are actively taking opioids at the time of revision TKA report significantly lower preoperative and postoperative outcome scores. These patients are more likely to have longer hospital stays. The apparent negative effect on patient reported outcomes after revision TKA provides clinically useful data for surgeons in engaging patients in a preoperative counseling regarding narcotic use prior to revision TKA to optimize outcomes.
\end{abstract}

received

August 31, 2020

accepted after revision

April 1, 2021

article published online

May 15, 2021 (c) 2021. Thieme. All rights reserved.

Thieme Medical Publishers, Inc., 333 Seventh Avenue, 18th Floor, New York, NY 10001, USA
DOI https://doi.org/ 10.1055/s-0041-1729966. ISSN 1538-8506. 
Over the past decade, the harmful effects of opioids have come into focus across the United States. Within the orthopaedic community, there has been a concerted effort to highlight and reduce opioid usage given their harmful effects. $^{1,2}$ While trends demonstrate improvement in prescribing practices, the opioid epidemic continues to plague the United States.

Preoperative opioid use has been associated with increased rates of prosthetic joint infection (PJI), all-cause revision, postoperative complications, readmission rates, and longer hospital stays in patients undergoing primary total joint arthroplasty (TJA). ${ }^{1-5}$ Despite this substantial body of literature on the effect of opioids on quality metrics, few studies have examined the impact opioid use patterns on patient-reported outcome measures (PROMs). ${ }^{1,6}$ Patientreported outcomes, such as the patient reported outcomes measurement information system short form (PROMIS SF) physical, mental, and SF 10A, are patient response surveys that provide a direct evaluation of patient opinion and functional outcomes following surgery. ${ }^{7,8}$ These tools are increasingly being studied in the arthroplasty setting to better understand patient-perceived effect of their hip and knee replacement. $^{9-11}$

The burden of revision total knee arthroplasty (TKA) is expected to increase between 78 and $182 \%$ by $2030 .{ }^{12}$ Opioid use is of particular concern in this population, as the percentage of opioid-naïve patients is lower among revision joint arthroplasty patients as compared with primary arthroplasty. ${ }^{1}$ The cause of this increased use has not been elucidated; however, prolonged opioid use after TKA and other major surgeries is likely a contributing factor. ${ }^{13}$ The impact of preoperative opioid use on PROM following revision TKA is important to elucidate as patients undergoing revision arthroplasty are already expected to experience reduced improvement in PROM as compared with primary arthroplasty. ${ }^{14}$ The aim of this investigation was to characterize the effect of preoperative narcotic use on PROMs following revision TKA. The authors' hypothesized that patients taking opioids preoperatively would experience reduced improvement postoperatively as compared with opioid-native patients.

\section{Materials and Methods}

\section{Patients}

The institutional review board (IRB) approved a retrospective review of all patients who underwent revision TKA who had also completed both pre- and postoperative PROMs. All revision surgeries were performed by a total of seven fellowship trained orthopaedic surgeons. The PROMs collected included PROMIS Global-10, the physical function short form 10A (SF 10A), and the knee disability and osteoarthritis outcome score physical function (KOOS-PS). ${ }^{15-17}$ The electronic medical record (EMR) was queried to identify patients who met these inclusion criteria. Age, laterality, sex, American Society of Anesthesiology (ASA) score, body mass index (BMI), comorbid psychiatric conditions, length of stay (LOS), PROM follow-up time, indication for surgery, and opioid use were all collected from the chart using hospital progress notes, clinic notes, or operative reports.

The type of opioid and dosage at the time of presentation for revision TKA was captured directly from the "medications prior to admission" section of the EMR which is reconcile on the day of presentation for surgery. Data collected on preoperative opioid use included the medication, dosage, and frequency. For comparison, all medications were converted to morphine milligram equivalents (MME's). ${ }^{1}$ Opioid medications were identified according to the CDC definitions of natural (i.e., morphine), semisynthetic (i.e., oxycodone), and synthetic (i.e., tramadol or methadone) opioids. Patients were thus divided into two cohorts: those who were and were not using opioids preoperative immediately prior to revision TKA.

\section{Cohort Matching}

To control for potential confounding variables, ${ }^{1}$ patients taking opioids preoperatively were matched $1: 2$ to nonusers on the basis of the propensity score using the nearestneighbor matching to investigate the true effect of opioid use on PROMs following revision TKA. The propensity score matching included the following parameters: age, gender, BMI, ASA score, depression or anxiety, as well as revision indications. A maximum difference of 0.2 was chosen for differences between propensity probabilities. ${ }^{18}$ A standardized mean difference for each covariate was utilized to examine the balance of covariate between both cohorts.

\section{Patient-Reported Outcome Measures}

The KOOS-PS, PROMIS SF physical, PROMIS SF mental, as well as physical SF 10A, scores were collected on average 4.3 days (2.7 days) prior to surgery and 12.5 months (8.5 months) following revision TKA. ${ }^{15,16}$ The minimally clinical important difference (MCID) was set as 10 points for KOOS-PS as determined by Nilsdotter et al. ${ }^{17}$ The MCID for the PROMIS SF physical, PROMIS SF mental, as well as physical SF 10A, were set to ranges of 4 to 6 and 3 to 5 respectively, as utilized by other studies in the literature. ${ }^{1,19}$

\section{Power Analysis}

A statistical power analysis was performed for sample size estimation to determine the number needed to detect a significant statistical difference between groups. The power analysis was performed based on data from a similarly designed study for primary TKA patients. ${ }^{5}$. With an $\alpha=0.05$, power $=0.80$, and the same sampling ratio, the projected sample size needed for this study is approximately 38 opioid users and 78 nonusers.

\section{Statistics}

To ensure that each cohort was appropriately matched, we performed paired two-tailed Student's $t$-test for continuous variables (age, BMI, LOS, and follow-up time), and Pearson's Chi-square test for categorical variables (gender, laterality, ASA score, presence of psychiatric or chronic pain diagnoses, disposition, and multimodal use). The KOOS-PS, PROMIS SF physical, PROMIS SF mental, and SF 10A scores were used to 
assess patient-reported outcomes both preoperatively and postoperatively at follow-up. Means and standard deviations of these scores were calculated, and two-tailed values of $p<0.05$ were considered statistically significant.

\section{Results}

\section{Patients}

Prior to propensity score matching, we identified 330 consecutive patients who underwent revision TKA and completed PROMs both preoperatively and postoperatively. Of these patients, 128 (39\%) were identified as preoperative opioid users, and $202(61 \%)$ were identified as nonusers. There was no difference between the cohorts with regard to gender, surgical laterality, age, BMI, comorbid psychiatric conditions, or PROM follow-up time (-Table 1). Patients reporting preoperative opioid use demonstrated a significantly higher ASA score (2.7 vs. $2.2 ; p<0.001)$. Preoperative opioid users also had longer average postoperative length of hospital stay (4.0 vs. 3.1 days; $p<0.001$ ).

Following propensity score matching, 297 propensity score-matched patients were included for analysis forming two cohorts as follows: (1) 99 patients who reported opioid use prior to revision TKA, and (2) 198 propensity scorematched patients who did not. There was no significant difference between both cohorts with regard to patient demographics and medical comorbidities (-Table 2). Mean length of stay (LOS) was longer for opioid users (4.1 days \pm 0.7 days) compared with the matched nonusers (3.3 days \pm 0.6 days; $p<0.001$; - Table 2 ).

\section{Patient Reported Outcome Measures}

The KOOS-PS, PROMIS SF physical, PROMIS SF mental, as well as physical SF $10 \mathrm{~A}$, scores were collected on average 4.3 days (2.7 days) prior to surgery and 12.5 months (8.5 months) following revision TKA. There was no significant difference in PROM follow-up time between the both cohorts ( - Table 2 ).

Both propensity score-matched cohorts demonstrated statistically and clinically significant improvements in PROMs scores following revision TKA ( - Table 3 ). Preoperatively, those patients who reported opioid use prior to surgery had significantly lower scores across all four PROMs: KOOS-PS (45.2 vs. 53.8; $p<0.001$ ), PROMIS SF physical (37.2 vs. $42.5 ; p<0.001$ ), PROMIS SF mental (44.2 vs. 51.3; $p<0.001)$, and physical SF $10 \mathrm{~A} \quad(34.1$ vs. 36.8; $p<0.001$; - Table 3 ). The lower preoperative scores for PROMIS SF physical (delta 5.3) and PROMIS SF mental (delta 7.1) were also clinically significant. These trends were mirrored when examining postoperative scores with preoperative opioid users reporting lower 207 scores: KOOS-PS (59.2 vs. 67.2; $p=0.003$ ), PROMIS SF physical (43.2 vs. 52.4; $p<0.001$ ), 208 PROMIS SF mental (47.5 vs. $58.9 ; p<0.001$ ) and physical SF $10 \mathrm{~A}$ ( 40.5 vs. $49.4 ; p<0.001$; - Table 3 ). The lower postoperative scores for PROMIS SF physical (delta 9.2), PROMIS SF mental (delta 11.4) and physical SF 10A (delta 8.9; - Table 3 ) were also clinically significant.

Table 1 Patient cohort characteristics prior to propensity score matching

\begin{tabular}{|c|c|c|c|}
\hline Characteristic & Opioid user $(n=128)$ & Nonopioid user $(n=202)$ & $p$-Value \\
\hline Female/male & $67 / 61$ & $109 / 93$ & 0.821 \\
\hline Left/right & $64 / 64$ & $103 / 99$ & 0.910 \\
\hline Age (y) & $66.1 \pm 9.3$ & $68.2 \pm 10.6$ & 0.063 \\
\hline BMI $\left(\mathrm{kg} / \mathrm{m}^{2}\right)$ & $32.8 \pm 5.9$ & $31.5 \pm 6.1$ & 0.056 \\
\hline \multicolumn{4}{|l|}{ ASA score } \\
\hline 1 & $4(3.13)$ & $10(4.95)$ & \\
\hline 2 & $62(48.44)$ & $130(64.36)$ & 0.014 \\
\hline 3 & $60(46.88)$ & $59(29.70)$ & \\
\hline 4 & $2(1.56)$ & $2(0.99)$ & \\
\hline Depression/anxiety/psychiatric & $36(28.13)$ & $53(26.24)$ & 0.705 \\
\hline Length of stay (d) & $4.0 \pm 0.6$ & $3.1 \pm 0.6$ & $<0.001$ \\
\hline \multicolumn{4}{|l|}{ Revision indication } \\
\hline Aseptic loosening & $47(37.7)$ & $93(46.0)$ & 0.17 \\
\hline PJI & $38(29.6)$ & $29(14.3)$ & $<0.01$ \\
\hline Instability & $19(14.8)$ & $31(15.3)$ & 0.79 \\
\hline Wear & $3(2.3)$ & $3(1.4)$ & 0.47 \\
\hline Periprosthetic fracture & $5(3.9)$ & $7(3.4)$ & 0.9 \\
\hline Stiffness & $16(12.5)$ & $34(16.8)$ & 0.51 \\
\hline PROM follow-up time (mo) & $12.2 \pm 8.2$ & $12.8 \pm 8.8$ & 0.519 \\
\hline
\end{tabular}

Abbreviations: ASA, American Society of Anesthesiology; BMI, body mass index; PJI, prosthetic joint infection; PROM, patient-reported outcome measure. Note: Values are presented as $n, n(\%)$, or mean \pm standard deviation. 
118 Impact of Preoperative Opioid Use on PROMs after Revision TKA Ingall et al.

Table 2 Patient cohort characteristics following propensity score matching

\begin{tabular}{|c|c|c|c|}
\hline Characteristic & Opioid user $(n=99)$ & Nonopioid user $(n=198)$ & $p$-Value \\
\hline Female/male & $52 / 47$ & $104 / 94$ & 0.667 \\
\hline Left/right & $48 / 51$ & $98 / 100$ & 0.632 \\
\hline Age (y) & $66.7 \pm 9.0$ & $68.4 \pm 10.4$ & 0.254 \\
\hline BMI $\left(\mathrm{kg} / \mathrm{m}^{2}\right)$ & $32.3 \pm 5.7$ & $31.7 \pm 6.2$ & 0.447 \\
\hline \multicolumn{4}{|l|}{ ASA score } \\
\hline 1 & $3(3.0)$ & $4(2.0)$ & \\
\hline 2 & $50(50.5)$ & $93(46.7)$ & 0.340 \\
\hline 3 & $45(45.5)$ & $101(50.7)$ & \\
\hline 4 & $1(1.0)$ & $1(0.6)$ & \\
\hline Depression/anxiety/psychiatric & $20(20.2)$ & $51(25.7)$ & 0.769 \\
\hline Length of stay (d) & $4.1 \pm 0.7$ & $3.3 \pm 0.6$ & $<0.001$ \\
\hline \multicolumn{4}{|l|}{ Revision indication } \\
\hline Aseptic loosening & $44(44.4)$ & $92(46.4)$ & 0.239 \\
\hline PJI & $21(21.2)$ & $28(14.1)$ & 0.131 \\
\hline Instability & $18(18.0)$ & $30(15.1)$ & 0.673 \\
\hline Wear & $2(2.0)$ & $3(1.5)$ & 0.804 \\
\hline Periprosthetic fracture & $3(3.0)$ & $6(3.0)$ & 0.987 \\
\hline Stiffness & $11(11.0)$ & $34(17.2)$ & 0.266 \\
\hline PROM follow-up time (mo) & $12.5 \pm 8.1$ & $12.7 \pm 8.5$ & 0.720 \\
\hline
\end{tabular}

Abbreviations: ASA, American Society of Anesthesiology; BMI, body mass index; PJI, prosthetic joint infection; PROM, patient-reported outcome measure.

Note: Values are presented as $n, n(\%)$, or mean \pm standard deviation.

Table 3 PROM scores for the propensity score matched cohorts

\begin{tabular}{|c|c|c|c|c|c|}
\hline PROM score & Opioid users & Nonusers & $p$-Value & Delta & MCID \\
\hline \multicolumn{6}{|l|}{ Preoperative } \\
\hline KOOS-PS & $45.2 \pm 15.8$ & $53.8 \pm 14.9$ & $<0.001$ & 8.6 & 10 \\
\hline PROMIS SF physical & $37.2 \pm 7.1$ & $42.5 \pm 8.3$ & $<0.001$ & 5.3 & $4-6$ \\
\hline PROMIS SF mental & $44.2 \pm 9.8$ & $51.3 \pm 9.0$ & $<0.001$ & 7.1 & $3-5$ \\
\hline Physical SF10A & $34.1 \pm 4.8$ & $36.8 \pm 6.1$ & $<0.001$ & 2.7 & $3-5$ \\
\hline \multicolumn{6}{|l|}{ Postoperative } \\
\hline KOOS-PS & $59.2 \pm 16.6$ & $67.2 \pm 13.6$ & 0.003 & 8.0 & 10 \\
\hline PROMIS SF physical & $43.2 \pm 7.9$ & $52.4 \pm 9.1$ & $<0.001$ & 9.2 & $4-6$ \\
\hline PROMIS SF mental & $47.5 \pm 8.5$ & $58.9 \pm 7.2$ & $<0.001$ & 11.4 & $3-5$ \\
\hline Physical SF 10A & $40.5 \pm 8.1$ & $49.4 \pm 9.8$ & $<0.001$ & 8.9 & $3-5$ \\
\hline
\end{tabular}

Abbreviations: KOOS-PS, knee disability and osteoarthritis outcome score physical function; MCID, minimally clinical important difference; PROM, patient-reported outcome measure; PROMIS SF, patient reported outcomes measurement information system short form.

Opioid users demonstrated a significantly lower absolute increase in PROMIS SF physical (6.0 vs. 9.9; $p=0.03$ ) and physical SF $10 \mathrm{~A}(6.4$ vs. $12.6 ; p<0.001)$ when compared with nonusers ( - Table 4).

\section{Discussion}

Opioid use has been associated with negative clinical and quality outcomes for patients after primary joint arthro- plasty. ${ }^{20-23}$ As revision TKA are expected to increase in the future, ${ }^{24}$ appropriate characterization of the impact of preoperative use is important to set expectations and preoperatively counsel patients. Moreover, as health care shifts toward a value-based model, identification of variables that can impact PROMs is of financial concern; particularly in a patient population, such as revision arthroplasty, which already represent a financial burden to the health care system. ${ }^{25}$ Wilson et al demonstrated increased complication rates and LOS in 
Table 4 Absolute improvement in patient-reported outcome measures after revision total knee arthroplasty

\begin{tabular}{|l|l|l|l|}
\hline PROM score & Opioid users & Nonusers & $p$-Value \\
\hline KOOS-PS & 14.0 & 13.4 & 0.93 \\
\hline $\begin{array}{l}\text { PROMIS SF } \\
\text { physical }\end{array}$ & 6.0 & 9.9 & 0.03 \\
\hline $\begin{array}{l}\text { PROMIS SF } \\
\text { mental }\end{array}$ & 7.3 & 7.6 & 0.67 \\
\hline Physical SF 10A & 6.4 & 12.6 & $<0.001$ \\
\hline
\end{tabular}

Abbreviations: KOOS-PS, knee disability and osteoarthritis outcome score physical function; PROM, patient-reported outcome measure; PROMIS SF, patient reported outcomes measurement information system short form.

patients who had received an opioid prescription in the year prior to their revision TKA. ${ }^{26}$ The data herein show that patients who report opioid use prior to revision TKA have significantly lower pre- and postoperative PROMs, when compared with propensity score-matched nonusers. Furthermore, preoperative opioid users experienced lower absolute increases in PROMIS SF physical and physical SF 10A.

Despite advances in multimodal pain regimen and the use of regional anesthetic techniques, opioid consumption has been demonstrated to be higher in revision TKA as compared with primary TKA. ${ }^{27}$ Pain control in those exposed chronic opioids preoperatively is more difficult to achieve. ${ }^{28}$ Thus, at higher doses, the negative side effects of opioids, such as urinary retention, ileus, nausea, and vomiting, may become more apparent. ${ }^{29}$

The negative impacts of preoperative opioid use on complications and patient morbidity following primary total hip arthroplasty (THA) and TKA are well known. ${ }^{5,20,21,30,31}$ Rozell et al identified that preoperative opioid use was an independent risk factor for needing intravenous rescue narcotics. ${ }^{20}$ This was correlated with increased risk of postoperative interventions such as fluid boluses, transfusions, and urinary catheterization. They also identified that preoperative opioid use more than doubled the risk of continued opioid use 3 months postoperatively. Sing et al specifically identified an association between use of long-acting narcotics preoperatively with postoperative complications, as well as increased likelihood of discharge to a rehab facility as opposed to home. ${ }^{22}$ A study following the Veterinary Affairs patients for 6 years after primary TKA found that preoperative opioid users were more likely to undergo early revision TKA, with an odds ratio of $1.40 .{ }^{24}$ Preoperative opioid use has been identified an independent risk factor for revision surgery ${ }^{32,33}$ and prolonged LOS. ${ }^{1,20}$ The findings on our study confirm the negative effect of preoperative opioid use on hospital LOS in the setting of revision TKA, which is of importance as revision TKA is associated with increased morbidity, mortality, and health care costs, when compared with primary TKA. ${ }^{1,3-5}$ In addition, the present study also demonstrates the negative effect of preoperative opioid use on PROMs.

A limited number of studies to date has reported on the functional outcomes of preoperative opioid users in the primary arthroplasty setting. Pivec et al reported on 54 patients who had used narcotics prior to primary THA. ${ }^{6}$ They evaluated the Harris Hip Scores and University of California Loss Angeles (UCLA) activity scores preoperatively and at subsequent postoperative follow-up visits. When compared with a nonnarcotic group, they found the preoperative opioid group had significantly worse Harris' hip scores at final follow-up. Zywiel et al found lower Knee Society scores at a mean of 3-year follow-up for patients using opioids at the time of primary TKA. ${ }^{2}$ Smith et al investigated The Western Ontario and McMaster Universities Arthritis Index (WOMAC) scores in patients undergoing primary TKA and found WOMAC scores were significantly higher in patients with preoperative opioid use. ${ }^{5}$ At 6-month follow-up, their mean 6-month WOMAC pain score reduction was compared with 33 in patients who did not use opioids preoperatively. Similarly, Bonner et al demonstrated significantly lower PROM scores for 76 patients with preoperative opioid use at 1-year following THA. ${ }^{1}$

The present study builds on this body of literature by investigating outcome measures in the revision TKA setting. Importantly, the KOOS-PS, PROMIS, and SF-10 tools are all patient-reported tools in contrast to physician-determined outcome measures such as the Harris hip score. The authors demonstrate concordant results with significantly lower preand postoperative KOOS-PS, PROMIS SF physical, SF mental, and SF 10A among opioid users, when compared with propensity score-matched nonopioid users. As revision surgery is associated with significant morbidity and mortality, the present study provides clinically useful data in engaging patients in a preoperative discussion about narcotic use prior to revision TKA to optimize outcomes.

\section{Strengths and Limitations}

The current findings should be interpreted in the context of its strengths and limitations. This is a retrospective study design with inherent limitations of reporting and recall bias. While the authors attempted to control for this in a matched cohort of nonopioid users, a prospectively designed study would obviate some of these inherent biases. PROMs tools, just as any survey tool, also have the potential for response/nonresponse bias, as they are voluntary surveys. Only patients who had completed both pre- and postoperative PROMS were eligible for inclusion. The strengths of this study include direct assessment of preoperative narcotic use on the day of revision TKA. In this manner, the authors are able to clearly identify a cohort of patients actively using this class of medication immediately prior to surgery as compared with other similar studies. $^{26}$

\section{Conclusion}

In conclusion, patients actively using opioids at the time of revision TKA report significantly lower preoperative and postoperative outcome scores when compared with propensity score-matched patients not actively taking narcotics. Furthermore, the study cohort demonstrated lower absolute 
increase in PROMIS SF physical and physical SF $10 \mathrm{~A}$ as compared with matched nonusers. Lastly, these patients were more likely to have longer LOS. The apparent negative effect on patient-reported outcomes after revision TKA provides clinically useful data for surgeons in engaging patients in a preoperative counseling regarding narcotic use prior to revision TKA to optimize outcomes.

\section{Conflict of Interest}

None declared.

\section{References}

1 Bonner BE, Castillo TN, Fitz DW, Zhao JZ, Klemt C, Kwon Y-M. Preoperative opioid use negatively affects patient-reported outcomes after primary total hip arthroplasty. J Am Acad Orthop Surg 2019;27(22):e1016-e1020

2 Zywiel MG, Stroh DA, Lee SY, Bonutti PM, Mont MA. Chronic opioid use prior to total knee arthroplasty. J Bone Joint Surg Am 2011;93(21):1988-1993

3 Crowninshield RD, Rosenberg AG, Sporer SM. Changing demographics of patients with total joint replacement. Clin Orthop Relat Res 2006;443(443):266-272

4 Hamilton DF, Howie CR, Burnett R, Simpson AHRW, Patton JT. Dealing with the predicted increase in demand for revision total knee arthroplasty: challenges, risks and opportunities. Bone Joint J 2015;97-B(06):723-728

5 Smith SR, Bido J, Collins JE, Yang H, Katz JN, Losina E. Impact of preoperative opioid use on total knee arthroplasty outcomes. J Bone Joint Surg Am 2017;99(10):803-808

6 Pivec R, Issa K, Naziri Q, Kapadia BH, Bonutti PM, Mont MA. Opioid use prior to total hip arthroplasty leads to worse clinical outcomes. Int Orthop 2014;38(06):1159-1165

7 Davis AM, Perruccio AV, Canizares M, et al. Comparative, validity and responsiveness of the HOOS-PS and KOOS-PS to the WOMAC physical function subscale in total joint replacement for osteoarthritis. Osteoarthritis Cartilage 2009;17(07):843-847

8 Davis AM, Perruccio AV, Canizares M, et al. The development of a short measure of physical function for hip OA HOOS-Physical Function Shortform (HOOS-PS): an OARSI/OMERACT initiative. Osteoarthritis Cartilage 2008;16(05):551-559

9 Ramkumar PN, Harris JD, Noble PC. Patient-reported outcome measures after total knee arthroplasty: a systematic review. Bone Joint Res 2015;4(07):120-127

10 Finch DJ, Martin BI, Franklin PD, Magder LS, Pellegrini VDJ Jr. PEPPER Investigators. Patient-reported outcomes following total hip arthroplasty: a multicenter comparison based on surgical approaches. J Arthroplasty 2020;35(04):1029-1035.e3

11 Stiegel KR, Lash JG, Peace AJ, Coleman MM, Harrington MA, Cahill CW. Early experience and results using patient-reported outcomes measurement information system scores in primary total hip and knee arthroplasty. J Arthroplasty 2019;34(10): 2313-2318

12 Kurtz SM, Lau E, Ong K, Zhao K, Kelly M, Bozic KJ. Future young patient demand for primary and revision joint replacement: national projections from 2010 to 2030. Clin Orthop Relat Res 2009;467(10):2606-2612

13 Politzer CS, Kildow BJ, Goltz DE, Green CL, Bolognesi MP, Seyler TM. Trends in opioid utilization before and after total knee arthroplasty. J Arthroplasty 2018;33(7S):S147-S153

14 Postler AE, Beyer F, Wegner T, et al. Patient-reported outcomes after revision surgery compared to primary total hip arthroplasty. Hip Int 2017;27(02):180-186
15 Cella D, Yount S, Rothrock N, et al; PROMIS Cooperative Group. The Patient-reported outcomes measurement information system (PROMIS): progress of an NIH roadmap cooperative group during its first two years. Med Care 2007;45(05, Suppl 1):S3-S11

16 Bohannon RW, DePasquale L. Physical Functioning Scale of the Short-Form (SF) 36: internal consistency and validity with older adults. J Geriatr Phys Ther 2010;33(01):16-18

17 Nilsdotter AK, Lohmander LS, Klässbo M, Roos EM. Hip disability and osteoarthritis outcome score (HOOS)-validity and responsiveness in total hip replacement. BMC Musculoskelet Disord 2003;4:10

18 Tan TL, Goswami K, Kheir MM, Xu C, Wang Q, Parvizi J. Surgical treatment of chronic periprosthetic joint infection: fate of spacer exchanges. J Arthroplasty 2019;34(09):2085-2090.e1

19 Yost KJ, Eton DT, Garcia SF, Cella D. Minimally important differences were estimated for six patient-reported outcomes measurement information system-cancer scales in advanced-stage cancer patients. J Clin Epidemiol 2011;64(05):507-516

20 Rozell JC, Courtney PM, Dattilo JR, Wu CH, Lee G-C. Preoperative opiate use independently predicts narcotic consumption and complications after total joint arthroplasty. J Arthroplasty 2017;32(09):2658-2662

21 Carpinelli RN, Otto RM. Strength training. Single versus multiple sets. Sports Med 1998;26(02):73-84

22 Sing DC, Barry JJ, Cheah JW, Vail TP, Hansen EN. Long-acting opioid use independently predicts perioperative complication in total joint arthroplasty. J Arthroplasty 2016;31(9, Suppl):170-174.e1

23 Ben-Ari A, Chansky H, Rozet I. Preoperative opioid use is associated with early revision after total knee arthroplasty: a study of male patients treated in the veterans affairs system. J Bone Joint Surg Am 2017;99(01):1-9

24 Kurtz S, Ong K, Lau E, Mowat F, Halpern M. Projections of primary and revision hip and knee arthroplasty in the United States from 2005 to 2030. J Bone Joint Surg Am 2007;89(04):780-785

25 Peterson J, Sodhi N, Khlopas A, et al. A comparison of relative value units in primary versus revision total knee arthroplasty. J Arthroplasty 2018;33(7S):S39-S42

26 Wilson JM, Farley KX, Bradbury TL, Erens GA, Guild GN. Preoperative opioid use is a risk factor for complication and increased healthcare utilization following revision total knee arthroplasty. Knee 2020;27(04):1121-1127

27 Bernstein J, Feng J, Mahure S, Schwarzkopf R, Long W. Revision total knee arthroplasty is associated with significantly higher opioid consumption as compared with primary total knee arthroplasty in the acute postoperative period. Arthroplast Today 2020; 6(02):172-175

28 Carroll IR, Angst MS, Clark JD. Management of perioperative pain in patients chronically consuming opioids. Reg Anesth Pain Med 2004;29(06):576-591

29 de Boer HD, Detriche O, Forget P. Opioid-related side effects: Postoperative ileus, urinary retention, nausea and vomiting, and shivering. A review of the literature. Best Pract Res Clin Anaesthesiol 2017;31(04):499-504

30 Halawi MJ, Vovos TJ, Green CL, Wellman SS, Attarian DE, Bolognesi MP. Opioid-based analgesia: impact on total joint arthroplasty. J Arthroplasty 2015;30(12):2360-2363

31 Zarling BJ, Sikora-Klak J, Bergum C, Markel DC. How do preoperative medications influence outcomes after total joint arthroplasty? J Arthroplasty 2017;32(9S):S259-S262

32 Bozic KJ, Lau E, Ong K, et al. Risk factors for early revision after primary total hip arthroplasty in Medicare patients. Clin Orthop Relat Res 2014;472(02):449-454

33 Roche M, Law TY, Sodhi N, et al. Incidence of drug abuse in revision total knee arthroplasty population. J Knee Surg 2018;31 (10):928-933 